\title{
Incidence and Severity of Depression Among Recovered African Americans with COVID-19-Associated Respiratory Failure
}

\author{
Titilope Olanipekun ${ }^{1,2}$ (D) Temidayo Abe ${ }^{2} \cdot$ Valery Effoe ${ }^{2,3} \cdot$ Gloria Westney $^{2,4} \cdot$ Richard Snyder $^{2,4}$
}

Received: 13 January 2021 / Revised: 25 March 2021 / Accepted: 29 March 2021 / Published online: 6 April 2021

(C) W. Montague Cobb-NMA Health Institute 2021

\begin{abstract}
Background Coronavirus disease (COVID-19) disproportionately affects African Americans, and they tend to experience more severe course and adverse outcomes. Using a simple and validated instrument of depression screening, we evaluated the incidence and severity of major depression among African American patients within 90 days of recovery from severe COVID-19-associated respiratory failure.

Methods African American patients hospitalized and treated with invasive mechanical ventilation for COVID-19-associated respiratory failure in the intensive care unit (ICU) of Grady Memorial Hospital, Atlanta, between April 1, 2020, and June 30, 2020, were screened for depression within 90 days of hospital discharge using the validated patient health questionnaires (PHQ-2) and PHQ-9.

Results A total of 73 patients completed the questionnaire. The median age was 52.5 years [IQR 44-65] and 65\% were males. The most common comorbidities were hypertension (66\%) and diabetes mellitus (51\%). Forty-four percent of the patients had a diagnosis of major depressive disorder (MDD) based on their PHQ-9 questionnaire responses. The incidence of MDD was higher among females $(69 \%, n=18 / 26)$ compared to males $(29 \%, n=14 / 47)$, in patients $>75$ years $(66 \%)$ and those with multiple comorbidities (45\%). Eighteen percent of the patients had moderate depression, while $15 \%$ and $22 \%$ had moderately severe and severe depression, respectively. Only $26 \%(n=7 / 27)$ of eligible patients were receiving treatment for depression at the time of this survey.

Conclusion The incidence of depression in a cohort of African American patients without prior psychiatric conditions who recovered from severe COVID-19 infection was 44\%. More than $70 \%$ of these patients were not receiving treatment for depression.
\end{abstract}

Keywords African Americans · COVID-19 · Depression · ICU · PHQ-9

\section{Introduction}

The mental health impact of COVID-19 infection from the novel severe acute respiratory syndrome coronavirus 2 (SARS-CoV-2) on its survivors has been reported in the literature [1]. According to surveys conducted during the ongoing

Titilope Olanipekun

titilope_olanipekun@teamhealth.com; toolanipekun@gmail.com

1 Department of Hospital Medicine, Covenant Health System, Knoxville, TN 37922, USA

2 Department of Internal Medicine, Morehouse School of Medicine, Atlanta, GA, USA

3 Division of Cardiology, Atlanta, GA, USA

4 Division of Pulmonary and Critical Care Medicine, Morehouse School of Medicine, Atlanta, GA, USA
COVID-19 pandemic, patients often suffer from anxiety, posttraumatic stress disorder, and trouble sleeping after recovery from COVID-19 infection [2-4].

COVID-19 disproportionately affects African Americans, and they tend to experience more severe course and adverse outcomes $[5,6]$. Also, they are more likely to have undiagnosed and untreated major depression which can negatively impact daily functioning and lead to a poor quality of life [7, 8]. Currently, there is paucity of data on the incidence of depression among African Americans who have recovered from COVID-19 infection particularly those who had severe infection requiring intensive care unit (ICU) hospitalization.

Using a simple and validated instrument of depression screening, we evaluated the incidence and severity of major depression among African American patients within 90 days of recovery from severe COVID-19 infection with associated respiratory failure. 


\section{Methods}

The study was approved by the Morehouse School of Medicine institutional review board, and verbal informed consent was obtained from the study participants. We identified African American patients with confirmed severe acute respiratory syndrome coronavirus 2 (SARS-CoV-2), the virus that causes COVID-19 infection, on polymerase chain reaction testing of a nasopharyngeal or tracheal sample. Those who were hospitalized and treated with invasive mechanical ventilation for respiratory failure at the Grady Memorial Hospital ICU, Atlanta, Georgia, between April 1, 2020, and June 30, 2020 , were included in the study. Patients who were still hospitalized by June 30, 2020, and those with previous history of psychiatric diagnosis including major depression were excluded. Data on sociodemographics and comorbidities were extracted from the electronic health record system (Epic 2019 version).

The Patient Health Questionnaire (PHQ)-2 and PHQ-9 (supplementary material) are used to reliably assess for depression and its severity in the outpatient and other medical care settings [9]. The PHQ-9 is a nine-item questionnaire, and the PHQ-2 includes the first two items of the PHQ-9. The PHQ-2 evaluates the frequency of depressed mood and anhedonia over the past 2 weeks as a "first step" in the screening process. The PHQ-9 is then administered to patients if the PHQ-2 is positive for depression to determine whether they meet criteria for a depressive disorder [9-11]. The PHQ-2 has a $97 \%$ sensitivity and $67 \%$ specificity in adults, whereas the PHQ-9 has a $61 \%$ sensitivity and $94 \%$ specificity in adults [9].

A team of two physicians trained on the depression screening tools administered the PHQ-9 over the phone to the study patients within 30 to 90 days of discharge from the hospital. Verbal informed consent was obtained from the patients prior to administering the questionnaire. Patients who responded "yes" to at least 5 questions in the two right columns (one of which corresponds to question \#1 or \#2) of the PHQ-9 questionnaire were considered to have an initial diagnosis of major depressive disorder (MDD) $[10,11]$. Other depression is diagnosed if there are 2-4 yes answers in the right columns of the PHQ-9 (one of which corresponds to question \#1 or \#2) [11].

To assess depression severity, all patients were categorized into none or minimal, mild, moderate, moderately severe, and severe based on their severity scores on a scale of $0-27[10$, 11]. Using descriptive analysis, we determined the association between the severity of depression and the ICU length of stay (LOS) and discharge disposition. Lastly, we determined the number of patients on treatment for depression as a percentage of the total eligible for treatment based on their PHQ-9 scores. Summary statistics were reported as medians and proportions for various sociodemographic and clinical characteristics of patients. Due to limited sample size and descriptive nature of the study, analysis for statistical significance was performed. Data analysis was performed using $\mathrm{R}$ version 3.6.3 (R Foundation).

\section{Results}

A total of 91 patients met the inclusive criteria, and $73 \mathrm{com}-$ pleted the questionnaire (response rate of $80 \%$ ). Table 1 describes the sociodemographic and clinical characteristics of the patients with their PHQ-9 score distribution. The median age of the participants was 52.5 years [IQR 44-65]. There were more male participants than females $(65 \%$ vs. $35 \%)$. Eighty-five percent of the patients had more than 1 comorbidity, and the most common comorbidities were hypertension $(66 \%)$ and diabetes mellitus (51\%). Forty-four percent of the patients had a diagnosis of MDD based on their responses on the PHQ-9 questionnaire. The incidence of MDD was higher among females $(69 \%, n=18 / 26)$ compared to males $(29 \%$, $n=14 / 47)$. Sixty percent $(n=18 / 30)$ of patients aged $55-74$ years, and $66 \%(n=4 / 6)$ of patients $>75$ years had PHQ-9 scores consistent with MDD. Among patients with multiple comorbidities, $45 \%(n=28 / 62)$ had a diagnosis of MDD compared to $36 \%(n=4 / 11)$ in patients with only one or no comorbidity. Comorbidities with the highest proportion of patients MDD diagnosis were cerebrovascular accident (62.5\%), obstructive sleep apnea (60\%), solid tumors (57.5\%), hypertension $(58.3 \%)$, and diabetes mellitus (51.4\%). Eighteen percent of the patients had moderate depression, while $15 \%$ and $22 \%$ had moderately severe and severe depression, respectively.

The median LOS of participants in the ICU was 10 days [IQR 4-18.5]. Among patients who spent 10 days or more in the ICU, 58\% had a PHQ-9 score reflecting MDD compared to $32.5 \%$ in those who spent less than 10 days. Seventy-five percent of the patients were discharged home, out of which $38 \%$ had a diagnosis of MDD (Table 2). Though only $8 \%$ of the patients were discharged to a long-term acute care facility (LTAC), MDD diagnosis was disproportionately higher $(67 \%, n=4 / 6)$ relative to those discharged home $(38.2 \%)$ or skilled nursing facilities (SNF) (58.3\%).

Figure 1 shows that $37 \%(n=27 / 73)$ of patients in the study had a PHQ-9 score of at least 14 and were therefore eligible for depression treatment using antidepressants, psychotherapy, or a combination of both [10]. However, only $26 \%(n=7 / 27)$ were on some form of depression treatment at the time of this survey. Two of the patients were on cognitive behavioral therapy (CBT), while 5 patients were treated with a combination of CBT and antidepressant medications - selective serotonin receptor inhibitors (SSRIs). The remaining 74\% said they had not been screened for depression since discharge from the hospital. Though the research team at the time of the survey implored all the study patients to follow up with their primary care 


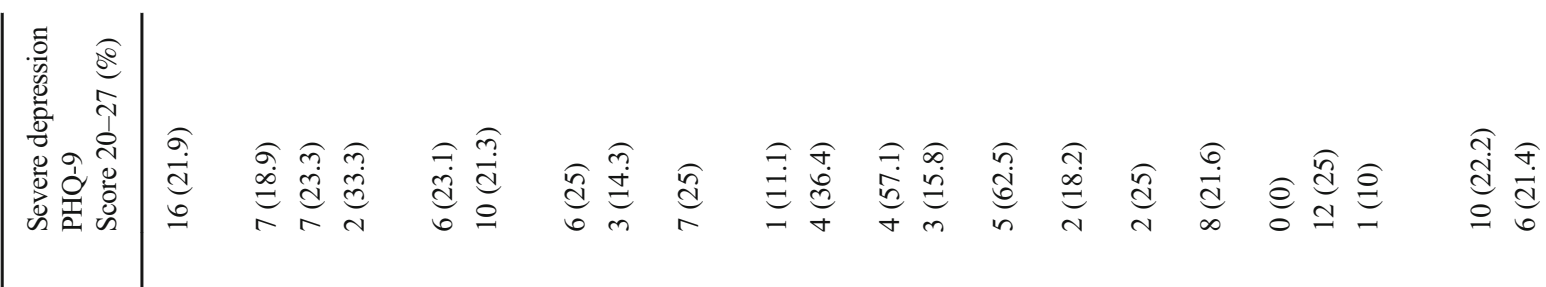

:

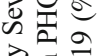

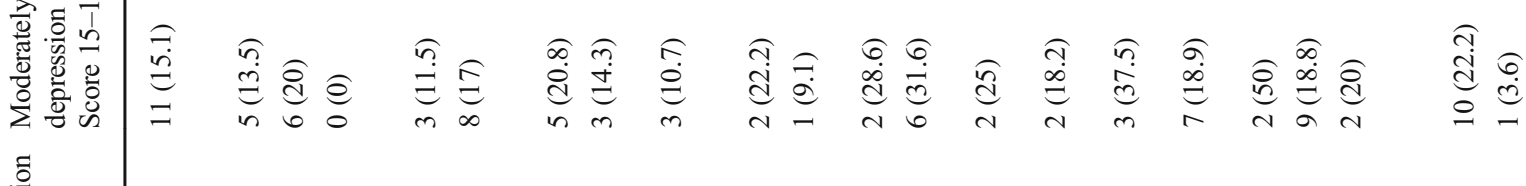

递

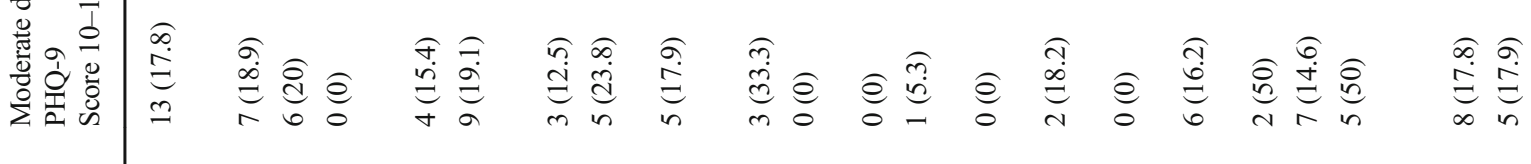

竞

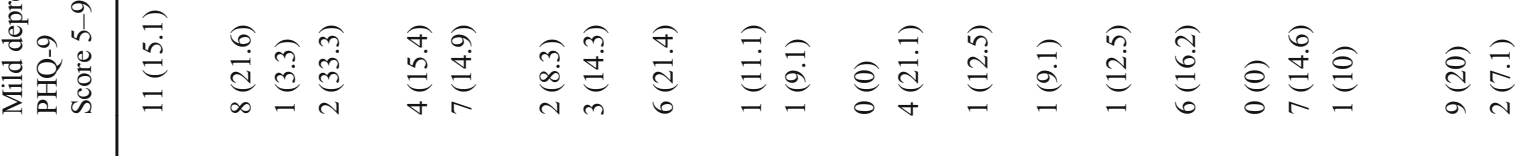

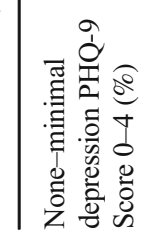

సิ

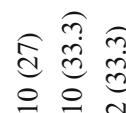

$0 \stackrel{2}{i} \approx$

है

สิก กิ

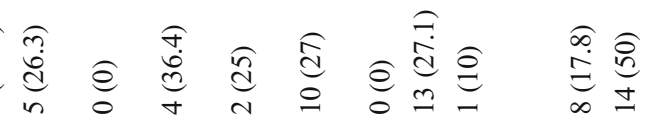

बิ

过

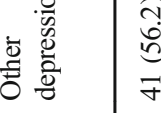

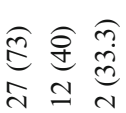

कृ

ह̂.

过

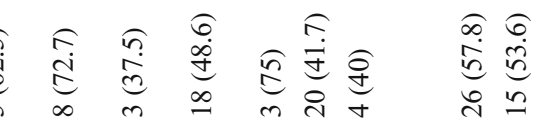

.

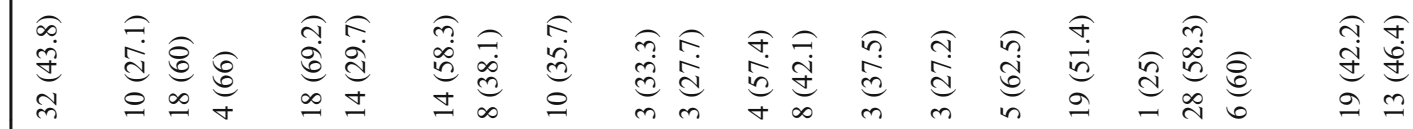

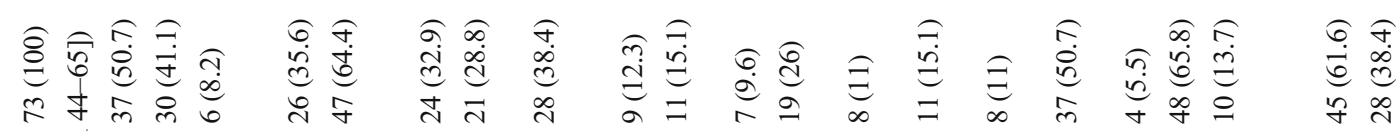

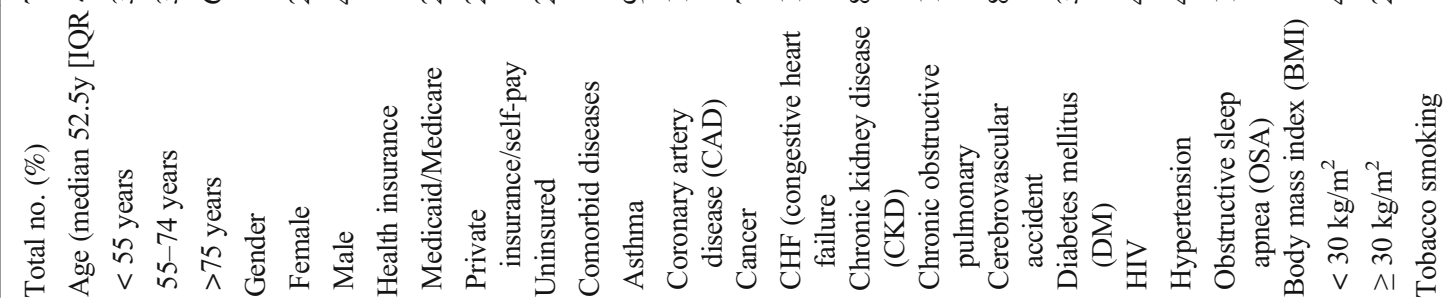




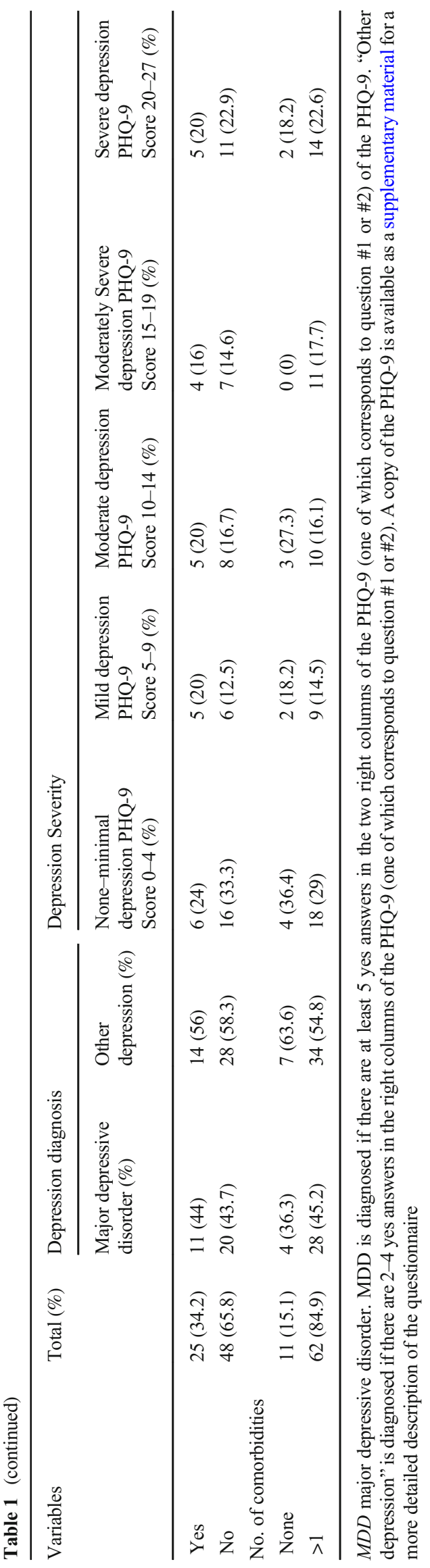

providers to be screened for depression and treatment, they particularly emphasized the need for urgent evaluation in patients with PHQ-9 score of at least 14 . No patient expressed homicidal or suicidal ideations (item 9 on the PHQ-9 questionnaire).

\section{Discussion}

Our study showed that at least 4 out of 10 African Americans without prior psychiatric illness, who were hospitalized in the ICU and received invasive mechanical ventilation for severe COVID-19 infection, will develop MDD within 90 days of discharge from the hospital. Notably, patients older than 55 years, females, patients with multiple pre-existing comorbidities, and those who spent 10 days or more in the ICU had higher rates of MDD diagnosis.

Depression is a component of the post-intensive care syndrome (PICS) and is common in ICU survivors. Studies have reported depression rates as high as $45 \%$ in patients with respiratory failure which is similar to what we found [12]. However, the rate of depression in our study was higher than what has been previously reported in patients who recovered from COVID-19 infection [1,3]. Psychiatric complications of SARS-CoV-2 infection have been attributed to the immune response to the virus itself, lingering clinical symptoms, and/ or psychosocial stressors from social isolation, concerns about infecting others, and social stigma [3]. It is important to point out that African Americans are uniquely at an additional risk of depression from socioeconomic challenges due to racism and inequitable access to resources and healthcare which may also have contributed to the higher rate of depression in our study [13].

Expectedly, patients discharged to LTAC facilities and SNF had disproportionately higher incidence of MDD compared to those discharged home. Patients discharged to extended care facilities (SNF and LTACs) are traditionally older, sicker, and more likely to have multiple comorbidities which are risk factors for MDD $[14,15]$. Studies show that up to $35 \%$ of residents in long-term care facilities may experience major depression [15]. However, depressive symptoms in patients in these facilities are often not recognized by healthcare providers [16]. Given that a significant proportion of patients with severe COVID-19 infection will reside in nursing facilities and LTACs following discharge from the hospital, it is imperative that physicians and nurses and ancillary staff at the nursing homes focus on routine screening of these patients for depression and treat accordingly.

It is quite concerning that $74 \%$ of patients in our study with moderate and moderately severe depression had not been evaluated by a medical professional and were not on any form of treatment. There is evidence in the literature to suggest that a significant proportion of African Americans have 
Table 2 Distribution of patients PHQ-9 scores by discharge disposition and length of hospital stay in the ICU and on invasive mechanical ventilation

\begin{tabular}{|c|c|c|c|c|c|c|c|}
\hline \multirow{2}{*}{$\begin{array}{l}\text { Length of stay (LOS) and } \\
\text { discharge disposition }\end{array}$} & \multirow[t]{2}{*}{ No $(\%)$} & \multirow[t]{2}{*}{ MDD } & \multicolumn{5}{|l|}{ Depression severity } \\
\hline & & & $\begin{array}{l}\text { None or minimal } \\
\text { depression PHQ-9 } \\
\text { Score } 0-4\end{array}$ & $\begin{array}{l}\text { Mild } \\
\text { depression } \\
\text { PHQ-9 } \\
\text { Score 5-9 }\end{array}$ & $\begin{array}{l}\text { Moderate } \\
\text { depression } \\
\text { PHQ-9 } \\
\text { Score } 10-14\end{array}$ & $\begin{array}{l}\text { Moderately severe } \\
\text { depression PHQ-9 } \\
\text { Score 15-19 }\end{array}$ & $\begin{array}{l}\text { Severe } \\
\text { depression } \\
\text { PHQ-9 } \\
\text { Score 20-27 }\end{array}$ \\
\hline Total no. $(\%)$ & $73(100)$ & $32(43.8)$ & $22(30.1)$ & $11(15.1)$ & $13(17.8)$ & $11(15.1)$ & $16(21.9)$ \\
\hline \multicolumn{8}{|l|}{ LOS } \\
\hline ICU $\operatorname{LOS}<10$ days & $40(54.8)$ & $13(32.5)$ & $14(35)$ & $10(25)$ & $5(12.5)$ & $6(15)$ & $5(12.5)$ \\
\hline ICU $\operatorname{LOS} \geq 10$ days & $33(45.2)$ & $19(57.5)$ & $8(24.2)$ & $1(3)$ & $8(24.2)$ & $5(15.2)$ & $11(33.3)$ \\
\hline $\begin{array}{l}\text { LOS on mechanical } \\
\text { ventilation }<10 \text { days }\end{array}$ & 45 (61.6) & $15(33.3)$ & $16(35.6)$ & $11(24.4)$ & $6(13.3)$ & $5(11.1)$ & $7(15.6)$ \\
\hline $\begin{array}{l}\text { LOS on mechanical } \\
\text { ventilation } \geq 10 \text { days } \\
\text { Discharge disposition }\end{array}$ & $28(38.4)$ & $17(60.7)$ & $6(21.4)$ & $0(0)$ & $7(25)$ & $6(21.4)$ & $9(32.1)$ \\
\hline Home & $55(75.3)$ & $21(38.2)$ & $19(34.5)$ & $11(20)$ & $9(16.4)$ & $5(9.1)$ & $11(20)$ \\
\hline SNF & $12(16.4)$ & $7(58.3)$ & $2(16.7)$ & $0(0)$ & $3(25)$ & $5(41.7)$ & $2(16.7)$ \\
\hline LTAC & $6(8.2)$ & $4(66.7)$ & $1(16.7)$ & $0(0)$ & $1(16.7)$ & $1(16.7)$ & $3(50)$ \\
\hline
\end{tabular}

$M D D$ major depressive disorder. MDD is considered if there are at least 5 yes answers in the two right columns (one of which corresponds to question \#1 or \#2) of the PHQ-9. A copy of the PHQ-9 is available as a supplementary material for a more detailed description of the questionnaire

undiagnosed depression and a relatively smaller proportion of those diagnosed with MDD go on to receive treatment compared to other ethnicities in the USA $[8,17]$. Additionally, African Americans and other racial minorities have less access to mental health services compared to whites and are more likely to receive poor quality care when treated [18]. This disparity in mental healthcare services is a deficit of the healthcare system that needs to be addressed.

Depression underdiagnosis and undertreatment among African Americans is rooted in societal stigma associated with mental illnesses, inadequate psychosocial support system, lack of information, and mistrust of the healthcare system [8]. Our study further underscores the need to prioritize the

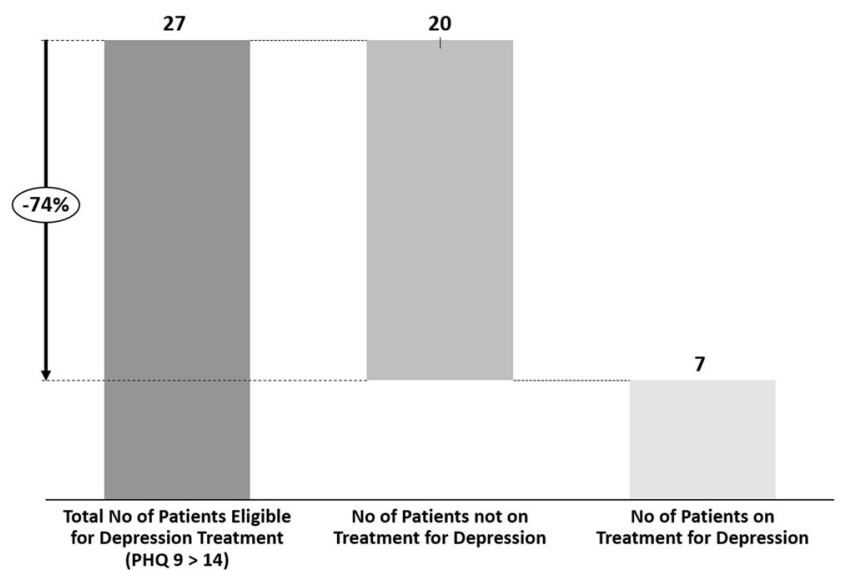

Fig. 1 Distribution of patients with PHQ-9 score $>14$ (moderately severe and severe depression, $n=27$ ) according to depression treatment status. Seventy-four percent $(n=20 / 27)$ of eligible patients were not receiving treatment for depression at the time of this survey mental health of COVID-19 survivors within the African American community and ensure timely and appropriated linkage to treatment services and interventions. The PHQ-9 questionnaire is sensitive in diagnosing MDD and easy to administer [10, 11, 19-22]. We recommend that medical providers utilize the PHQ-9 to screen COVID-19 survivors for depression during their post-ICU follow-up visits and manage appropriately.

Our study has some limitations. It is cross-sectional in nature, and causality cannot be implied. Also, the findings may not be generalizable to other ethnic groups. The sample size is relatively small, and this is a single center study; therefore, larger and multicenter prospective studies may be needed to further evaluate contextual factors that likely predispose COVID-19 patients to new psychiatric conditions.

\section{Conclusion}

The incidence of depression in a cohort of African American patients without prior psychiatric conditions who recovered from severe COVID-19 infection was $44 \%$. More than $70 \%$ of these patients were not receiving treatment for depression. Given the significantly high burden of COVID-19 infection in the African American community and the debilitating effect of depression, it is important for clinicians to assess for depression during post-ICU discharge follow-up for severe COVID19 infection-associated respiratory failure. Additionally, public health efforts should be intensified to help African 
Americans recognize the features of depression after COVID19 infection and seek medical evaluation early.

Supplementary Information The online version contains supplementary material available at https://doi.org/10.1007/s40615-021-01034-3.

Author Contribution All authors contributed to the writing of the manuscript.

\section{Declarations}

Ethics Approval This study was approved by the Morehouse School of Medicine Institutional Review Board (IRB).

Conflict of interest The authors declare no competing interests.

\section{References}

1. Taquet M, Luciano S, Geddes JR, Harrison PJ. Bidirectional associations between COVID-19 and psychiatric disorder: retrospective cohort studies of 62354 COVID-19 cases in the USA. Lancet Psychiatry. 2020.

2. Halpin SJ, McIvor C, Whyatt G, Adams A, Harvey O, McLean L, et al. Postdischarge symptoms and rehabilitation needs in survivors of COVID-19 infection: a cross-sectional evaluation. J Med Virol. 2020.

3. Mazza MG, De Lorenzo R, Conte C, Poletti S, Vai B, Bollettini I, et al. Anxiety and depression in COVID-19 survivors: role of inflammatory and clinical predictors. Brain Behav Immun. 2020;89: 594-600.

4. Zhang J, Lu H, Zeng H, Zhang S, Du Q, Jiang T, et al. The differential psychological distress of populations affected by the COVID19 pandemic. Brain Behav Immun. 2020:49-50.

5. Yancy CW. COVID-19 and African Americans. JAMA - J Am Med Assoc. 2020:1891-2.

6. Suleyman G, Fadel RA, Malette KM, Hammond C, Abdulla H, Entz A, et al. Clinical characteristics and morbidity associated with coronavirus disease 2019 in a series of patients in Metropolitan Detroit. JAMA Netw Open. 2020:e2012270.

7. Williams DR, González HM, Neighbors H, Nesse R, Abelson JM, Sweetman J, et al. Prevalence and distribution of major depressive disorder in African Americans, Caribbean blacks, and nonHispanic whites: results from the National Survey of American Life. Arch Gen Psychiatry. 2007;64:305-15.

8. Sohail Z, Bailey RK, Richie WD. Misconceptions of depression in African Americans. In: Front. Psychiatry; 2014.
9. O’Byrne P, Jacob JD. Screening for depression: review of the Patient Health Questionnaire-9 for nurse practitioners. J Am Assoc Nurse Pract. 2018;30:406-11.

10. Kroenke K, Spitzer RL, Williams JBW. The PHQ-9: Validity of a brief depression severity measure. J Gen Intern Med. 2001;16:60613 .

11. Kroenke K, Spitzer RL. The PHQ-9: a new depression diagnostic and severity measure. Psychiatr Ann. 2002:509-15.

12. Battle C, James K, Temblett P. Depression following critical illness: analysis of incidence and risk factors. J Intens Care Soc. 2015;16: $105-8$.

13. Pieterse AL, Todd NR, Neville HA, Carter RT. Perceived racism and mental health among black american adults: a meta-analytic review. J Couns Psychol. 2012;59:1-9.

14. Edgerton JR, Herbert MA, Mahoney C, Armstrong D, Dewey TM, Holper E, et al. Long-term fate of patients discharged to extended care facilities after cardiovascular surgery. Ann Thorac Surg. 2013;96:871-7.

15. Thakur M, Blazer DG. Depression in Long-Term Care. J Am Med Dir Assoc. 2008:82-7.

16. Smith M, Stolder ME, Jaggers B, Liu MF, Haedtke C. Depression training in nursing homes: lessons learned from a pilot study. Issues Ment Health Nurs. 2013;34:90-102.

17. Bailey RK, Patel M, Barker NC, Ali S, Jabeen S. Major depressive disorder in the African American population. J Natl Med Assoc. 2011:548-59.

18. McGuire TG, Miranda J. New evidence regarding racial and ethnic disparities in mental health: Policy implications. Health Aff. 2008;27:393-403.

19. Pinto-Meza A, Serrano-Blanco A, Peñarrubia MT, Blanco E, Haro JM. Assessing depression in primary care with the PHQ-9: can it be carried out over the telephone? J Gen Intern Med. 2005.

20. Zhang YL, Liang W, Chen ZM, Zhang HM, Zhang JH, Weng XQ, et al. Validity and reliability of patient health questionnaire- 9 and patient health questionnaire-2 to screen for depression among college students in China. Asia Pac Psychiatry. 2013;5:268-75.

21. De Man-Van Ginkel JM, Gooskens F, Schepers VPM, Schuurmans MJ, Lindeman E, Hafsteinsdóttir TB. Screening for poststroke depression using the patient health questionnaire. Nurs Res. 2012;61: 333-41.

22. Cameron IM, Crawford JR, Lawton K, Reid IC. Psychometric comparison of PHQ-9 and HADS for measuring depression severity in primary care. Br J Gen Pract. 2008;58:32-6.

Publisher's Note Springer Nature remains neutral with regard to jurisdictional claims in published maps and institutional affiliations. 